\title{
Criminologie
}

\section{Le public et la violence ; l'analyse des rapports des commissions d'enquête}

\section{André Normandeau}

Volume 12, numéro 1, 1979

Justice et public

URI : https://id.erudit.org/iderudit/017102ar

DOI : https://doi.org/10.7202/017102ar

Aller au sommaire du numéro

Éditeur(s)

Les Presses de l'Université de Montréal

ISSN

0316-0041 (imprimé)

1492-1367 (numérique)

Découvrir la revue

Citer ce compte rendu

Normandeau, A. (1979). Compte rendu de [Le public et la violence ; l'analyse des rapports des commissions d'enquête]. Criminologie, 12(1), 81-88.

https://doi.org/10.7202/017102ar d'utilisation que vous pouvez consulter en ligne.

https://apropos.erudit.org/fr/usagers/politique-dutilisation/ 


\section{LE PUBLIC ET LA VIOLENCE ; \\ L'ANALYSE DES RAPPORTS DES COMMISSIONS D'ENQUETE}

André Normandeau

Le thème de la violence $a$ été abondamment (peut-être trop !) traité sous toutes ses facettes au cours des deux dernières décennies. De grands reportages ont paru sur le sujet à la télévision et dans les journaux quotidiens à grand tirage. Des romans, des écrits de philosophes, des « études sérieuses \$ ou des recherches de sciences humaines ont investigué ce phénomène de fond en comble.

Mais récemment, coup sur coup, deux Commissions d'enquête, qui ont eu un très large écho dans notre milieu, ont refait le pèlerinage aux \&sources > de la violence et ont essayé \&une nouvelle fois * de jeter un éclairage \& neuf \& sur ce sujet controversé. Il s'agit :

dU RAPPORT DU COMITE D'ETUDES SUR LA VIOLENCE, LA CRIMINALITE ET LA DELINQUANCE, présidé par Alain PEYREFITTE (qui fut nommé en cours de route ministre de la Justice).

Paris : La documentation française, 1977, 2 tomes et 8 volumes.

du RAPPORT DE LA COMMISSION ROYALE SUR LA VIOLENCE DANS L'INDUSTRIE DES COMMUNICATIONS, présidé par Judith LAMARSH (ancien ministre fédéral à Ottawa).

Toronto : Imprimeur de la Reine, 1977, 7 volumes en langue anglaise et le volume $I$ en langue française.

Ces rapports, évidemment, traitent de plusieurs sujets apparentés au phénomène de la violence, comme l'avait d'ailleurs fait il y a près de dix ans, à la suite des assassinats de Robert Kennedy et Martin Luther King, la COMMISSION NATIONALE (américaine) SUR LES CAUSES ET LA PRÉVENTION DE LA VIOLENCE, présidée par Milton EISENHOWER (Washington (D.C.), U.S., G.P.O., 1969, 13 volumes)

Les aspects politiques, économiques, sociaux et culturels de la violence sont passés en revue; les incidences biophysiques et anthropologiques ainsi que les composantes sociologiques et psychologiques sont décrites minutieusement, même si l'on doit 
avouer que ces « vols à vue d'oiseau 》 nous laissent souvent « sur notre faim».

Quoi qu'il en soit, nous n'avons pas l'intention de taxer la bonne volonté du lecteur de la revue CRIMINOLOGIE et de lui imposer une autre recension « panoramique » de ces rapports.

Nous avons l'intention plutôt, en continuité avec le thème du présent numéro de la revue, qui est axé sur l'opinion publique et la justice, d'attirer brièvement l'attention du lecteur-sur les enquêtes d'opinion sur la violence faites au nom de ces commissions.

\section{L'ÉTUDE AMÉRICAINE}

La recherche américaine, intitulée : "The American Public Looks at Violence (Rapport Eisenhower, 1969, volume 9, p. 341-370 et p. 503-517) est relativement \& simple », pour ne pas dire «simpliste ». Il s'agit de l'enquête d'opinion classique et traditionnelle «à la Gallup » réalisée par Louis Harris et associés.

L'échantillon « aréolaire » est constitué de 1176 adultes et de 496 adolescents (14 à 18 ans). Les entrevues couvrent trois secteurs principaux : a) les normes qui sont associées à la violence; b) l'étendue et la nature des expériences de violence de la personne interrogée; c) ses habitudes et ses préférences en matière de communications et de télévision.

Au sujet des \& normes », quatre caractéristiques se dégagent : (1) l'utilisation de la violence, indépendamment de sa gravité, n'est jamais approuvée de façon inconditionnelle ; (2) la violence * légère » est approuvée, sauf à l'intérieur des relations époux-épouse ; (3) cette violence permise est liée à la punition et au contrôle des enfants ou des étudiants par les parents et les professeurs, à l'autodéfense et à la protection de la propriété et de ses proches, à certaines manifestations de masculinité pour les adolescents et les hommes, et à l'autodéfense et l'application de la loi par les policiers ; (4) une violence beaucoup plus grave est en principe approuvée également lorsqu'il s'agit du travail des policiers.

Au sujet des \& expériences » de violence, la recherche établit un lien entre ceux qui avouent utiliser la violence et ceux qui approuvent l'utilisation de la violence. L'hypothèse ici serait que 
les normes sont directement liées aux expériences. Les auteurs n'essaient pas d'établir un lien causal parce que, disent-ils, personne ne sait «qui influence qui » en premier.

Au sujet de la télévision, en particulier, les hypothèses suivantes sont soulevées : est-ce que l'écoute intensive de la télévision augmente la probabilité d'un comportement violent? Est-ce que les normes projetées à la télévision, par des émissions violentes, affectent les normes des auditeurs dans le sens de cette probabilité ? Les réponses semblent être négatives. Les normes exposées à la télévision sont en contradiction avec celles épousées par la majorité des Américains. Le contraste est particulièrement saisissant si nous distinguons la violence approuvée et non approuvée. Pour les personnes interrogées, la légitimité et la légalité sont nécessaires pour justifier la violence, ce qui n'est pas le cas souvent des émissions de violence à la télévision où la violence est souvent utilisée sans restriction afin d'atteindre le but individuel recherché.

Il s'agit ici de la partie la plus faible de l'étude américaine et les auteurs, à notre avis, basent leurs interprétations plus sur le sens commun que sur des faits. Quoi qu'il en soit, signalons que des recherches plus sophistiquées, à la fois sur les plans théorique et méthodologique, ont été réalisées depuis ce temps aux ÉtatsUnis, et le lecteur intéressé est invité à s'y référer (par exemple, le livre de Monica Blumenthal et alii, Justifying Violence : Attitudes of American Men, Michigan, ISR., 1972).

\section{L'ÉTUDE FRANÇAISE}

La recherche française, intitulée : *Violence » (Rapport Peyrefitte, 1977, volume I, p. 5-101) est une étude réalisée sur l'image de la violence et les comportements violents dans la population française par l'Institut français d'opinion publique (I.F.O.P.).

L'échantillon (de nature technique «inconnue dans le texte) est de 1500 personnes « représentatif » de la population française âgée de 18 ans et plus.

Compte tenu qu'il s'agit d'une recherche commanditée par le comité gouvernemental qui a dû, selon l'usage, demander une réalisation \& rapide \ de l'enquête, nous considérons que la recherche de l'I.F.O.P. a beaucoup de mérite. En effet, l'utilisation 
d'une «typologie des résultats », par exemple, réussit à mieux faire parler les données que les * tris simples \& à la Gallup... ou à la Harris.

D'emblée, une constatation intéressante de la recherche s'impose : une large majorité de la population française partage aujourd'hui le sentiment très vif d'une montée de la violence. Non seulement plus de $80 \%$ des Français s'accordent pour penser qu'ils vivent une époque de violence, mais les deux tiers d'entre eux estiment que les comportements violents sont aujourd'hui plus répandus que dans le passé proche (15 ans) ou lointain (1900). Le sentiment alarmiste n'est cependant pas aussi répandu dans tous les milieux. On constate en particulier que les plus jeunes (moins de 20 ans) et les personnes qui ont un niveau d'études supérieur résistent davantage à l'idée dominante de la montée de la violence et au sentiment collectif d'une insécurité grandissante.

On se trouve cependant en face d'un phénomène d'opinion d'une amplitude peu commune et qui concerne toutes les catégories sociales. En particulier le clivage politique a peu d'effet et les préférences pour l'un ou l'autre camp n'entraînent pas d'opinion différente dans l'estimation du niveau actuel de la violence en France.

Il n'y a pas réellement polarisation sur une forme criminelle précise, mais toujours l'impression d'une aggravation de la criminalité sous toutes ses formes.

La peur de la jeunesse délinquante s'aggrave très nettement au fur et à mesure qu'on avance dans les catégories les plus âgées. De plus, de façon générale, beaucoup jugent que la politique en France s'accompagne de violence.

La majorité de ceux qui vivent dans le sentiment alarmiste d'un développement de la criminalité croient à l'exemplarité d'un système de sanctions sociales et pénales sévères.

Soulignons au passage les résultats des typologies des auteurs. Dans le premier cas, celui des * groupes d'opinion \$, il y a : A) les alarmistes-répressifs, dont les \& impressionnables $>(18 \%)$, les * durs $\gg(20 \%)$ et les \& moralisants * $(18 \%)$; B) les * militants * $(17 \%)$, et les «modérés » $(27 \%)$. Dans le second cas, celui des «groupes de comportements 》, il y a les «agressifs 》 $(15 \%)$, les « répressifs > $(17 \%)$, les «pacifiques > $(20 \%)$, les 
¿ violents > $(7 \%)$, les < réservés * $(11 \%)$ et les \& réalistes * (30\%).

Mentionnons, finalement, que l'I.F.O.P. a également réalisé une autre étude digne d'intérêt sur le prénomène spécifique de la * Télévision et Violence > (idem, p. 103-152). Il s'agit d'une étude d'incidence sur le public d'une émission \& violente, à la télévision. L'émission choisie était le film bien connu L'Incident. Les réactions des téléspectateurs ont été recueillies par une technique qualitative (le guide d'entretien) qui leur a permis de s'exprimer très librement sur la façon dont ils ont e vécu > ce film et sur l'analyse qu'ils en ont faite. Les interviews ont été réalisées le lendemain même de l'émission.

Tout compte fait, les deux «petites > études de l'I.F.O.P. sont intéressantes et de qualité scientifique, même si nous constatons qu'une équipe de recherche comme celle du Service d'études pénales et criminologiques (S.E.P.C.) du Ministère de la Justice (Paris, France) fait des travaux plus approfondis sur les représentations, les images, les attitudes et les opinions de la population française. Évidemment, le S.E.P.C. a « le temps de bien faire 》 puisqu'il n'est pas « pressé » par le calendrier d'un comité gouvernemental, tel le Comité Peyrefitte.

\section{L'ÉTUDE CANADIENNE}

Le rapport de la Commission canadienne (Rapport Lamarsh, 1977) s'inscrit dans un cadre à la fois plus étroit et plus vaste. Plus étroit parce que son intérêt était directement lié aux phénomènes de violence dans l'industrie des communications, et en particulier à la télévision. Plus vaste parce que, sur le thème spécifique *Violence et Télévision », la Commission a fouillé à fond cette relation particulière.

Dans cette perspective, les recherches effectuées pour la Commission ont utilisé de façon très limitée le sondage d'opinion sur la violence. En fait, les travaux de recherches ont utilisé surtout la technique d'analyse de contenu et certains instruments de psychologie expérimentale. C'est ainsi que les « habitudes d'écoute $>$ des téléspectateurs canadiens ont été scrutées a à la loupe typologique > (pourcentage et minutage des émissions de violence, d'amour, d'information...). Il est intéressant de constater que les Québécois francophones sont soumis à deux fois moins de 
« temps-violence $\gg$ que les Canadiens anglophones, surtout attribuable au fait que les Québécois produisent eux-mêmes plusieurs émissions alors que les Canadiens importent beaucoup plus d'émissions 《 violentes 》 américaines.

Les quelques études d'opinion et d'attitude méritent toutefois notre attention, encore que le lecteur intéressé à la sociologie de la violence sera déçu car les 5 études spécifiques sur le sujet sont d'orientation nettement psychologique.

Ainsi, une étude réalisée à Toronto (volume 5, p. 171 et suiv.) s'intéresse aux media de communications et aux * perceptions de la violence \ de la part de 408 personnes interrogées. Certaines personnes étaient des résidents de quartiers à faible taux de criminalité, d'autres à taux élevé de criminalité. L'étude est fort intéressante sur le plan qualitatif (malgré le nombre de personnes interrogées, il ne s'agit pas d'un échantillon représentatif).

Une autre étude faite également à Toronto (volume 6, p. 18 et suiv.) traite de la \& vulnérabilité aux effets des media \$. Par entrevue et questionnaire auprès d'un échantillon non représentatif de 339 enfants (6 à 17 ans) et de leurs parents, les auteurs établissent une typologie psychologique d'agressivité (voir le questionnaire, p. 109-123). Dans le même sens, mais avec un intérêt spécifique sur les relations * violence, media et troubles mentaux 》, et avec un échantillon non représentatif de 475 personnes, à Toronto, une autre étude (volume 6, p. 162 et suiv.) utilise des grilles d'attitudes plus complexes (agressivité, tolérance, anxiété, victimisation...). Les mêmes instruments ont été subséquemment utilisés (avec adaptation) auprès d'un échantillon non représentatif de 300 personnes hospitalisées (maladie mentale) ou emprisonnées en Ontario (volume 6, p. 233 et suiv.).

Enfin, une étude réalisée dans une petite ville, Saskatoon, auprès d'un échantillon non représentatif de 315 personnes, a permis de retracer les perceptions des téléspectateurs à certaines émissions de télévision (volume 6, p. 284 et suiv.).

Notre jugement global sur les recherches d'opinion effectuées pour la Commission Lamarsh est relativement négatif.

En effet, malgré l'intérêt des autres parties du rapport, ces études spécifiques sont décevantes tant sur le plan méthodologique 
(l'échantillon non représentatif, par exemple) que sur le plan des résultats et de leurs analyses, nettement trop * psychologisantes ».

Le lecteur pourrait se consoler (ou se désoler) en lisant la définition «éléphantesque » de la violence retenue par la Commission Lamarsh. Et je cite (volume 1, p. 1, en anglais, car la version française n'est pas encore disponible $-s i c)$.

The Nature of Violence

Violence is action which intrudes painfully or harmfully into the physical, psychological or social well-being of persons or groups.

Violence or its effect may range from trivial to catastrophic. Violence may be obvious or subtle.

It may arrive naturally or by human design.

Violence may take place against persons or against property. It may be justified or unjustified, or justified by some standards and not by others.

It may be real or symbolic.

Violence may be sudden or gradual.

The Nature of Media Violence

Violence depicted in film, television, sound, print or live performance is not necessarily the same as violence in real life.

Things not violent in reality may be violent in their portrayal. Violence presented in the media may reach large numbers of people, whereas real violence may not.

The media may use many artificial devices to lessen or to amplify its emotional and social effects.

Violence depicted may do harm the original violence may not have done - or it may have no impact at all.

\section{CONCLUSIONS}

Évidemment, les enquêtes d'opinion faites pour des Commissions d'enquête sur la violence, même si elles sont intéressantes pour le chercheur spécialisé dans ce domaine, ne justifient pas par elles-mêmes la valeur et le coût de telles Commissions. Comme le mentionnait récemment un collègue (M. Jean-Paul Brodeur) en parlant des Commissions d'enquête sur la police et la moralité, trois thèses relatives aux Commissions d'enquête peuvent être énoncées : 1) les Commissions d'enquête constituent un processus à la fois répétitif ; 2) rétrospectif et 3 ) dont les retombées sur la situation à l'origine de leur existence sont très faibles. Leur fonction politique est de produire pour la population l'apparence du changement politique et social. 
Dans cette perspective, le criminologue * citoyen > est justifié d'être relativement sceptique à l'égard de l'impact réel des Commissions d'enquête. Par contre, le criminologue * chercheur * profite souvent de ces Commissions pour entreprendre des recherches (d'opinion... ou autres, d'ailleurs) qui ne pourraient être faites autrement et dont les résultats sont utiles à l'avancement de la science criminologique, sur les plans méthodologique et théorique, et, nous l'espérons, sur le plan d'une certaine pratique de politique criminelle. En effet, l'emploi du sondage devient une des règles d'un «bon gouvernement $\gg$. Il apporte, ce que ne peuvent faire le recensement ou le référendum, trop pesants, rares et généraux, le \& mesurage de l'opinion sur des questions vitales de l'actualité ». Quels sont les problèmes qui préoccupent les citoyens ? Comment voient-ils le système de justice en matière criminelle et pénale ? Comment jugent-ils l'action de la police, des tribunaux, du système correctionnel ?... Le chercheur peut ainsi saisir ce que les gens savent sur divers problèmes du jour et établir un rapport entre d'une part la qualité et la quantité de leur information et, d'autre part, leurs opinions. La détection des \& zones d'ignorance s devrait permettre dans chaque cas de mettre en place des procédures d'information. Une nouvelle consultation donnerait alors les réactions du public «informé ». Ce dialogue triangulaire entre l'opinion, le spécialiste des sondages et le politique peut faire véritablement de ce mode d'investigation l'instrument de la démocratie.

Dans la gestion des affaires publiques comme dans l'entreprise, les impressions vagues, les intuitions personnelles ou les suppositions gratuites ne suffisent pas à rendre compte des situations réelles. Le recours au sondage sur la justice, la violence (ou autres sujets d'ailleurs) est corrélatif au moins d'une volonté de clarification.

Est-ce une * rationalisation > de chercheur?

$\mathrm{Au}$ lecteur, au politicien, au fonctionnaire, au praticien ou au contribuable (« payeur de taxes ») d'en juger. 